\title{
Mean and turbulence measurements in the boundary layer and wake of a symmetric aerofoil
}

\author{
K.S.Hebbar
}

Aerodynamics Division, National Aeronautical Laboratory, Bangalore- 560 017, India

\begin{abstract}
Detailed measurements of two-dimensional profiles of static pressure, mean velocity, turbulence intensity and Reynolds shear stress were carried out with conventional pressure probes and hot wire probes at preselected streamwise stations in the boundary layer and wake of a $12.5 \%$ thick, $600 \mathrm{~mm}$ chord twodimensional symmetric aerofoil mounted at zero incidence in a low speed wind tunnel. The chord Reynolds number was one million and the wake measurements extended up to three chord lengths (or nearly 660 trailing edge momentum thicknesses) downstream of the trailing edge. The data indicate rapid interaction of the wall layers immediately behind the trailing edge, leading to significant changes in the flow parameters close to the trailing edge. The relaxation of the wake is preceded by initial 'overshoot' in the streamwise profiles of mean-flow parameters and peak values of turbulence components. Further growth of the wake towards similarity/equilibriunt is discussed.
\end{abstract}

List of symbols

\begin{tabular}{|c|c|}
\hline$B$ & half-wake width [value of $y$ where $U=\frac{1}{2}\left(U_{\infty}+U_{y=0}\right)$ ] \\
\hline$c$ & aerofoil chord length \\
\hline$H$ & shape parameter \\
\hline$T K E$ & turbulent kinetic energy \\
\hline$u, v, w$ & $\begin{array}{l}\text { turbulence fluctuations in } x, y, z \text { directions, respectively } \\
\text { (Fig. 1) }\end{array}$ \\
\hline$U, V, W$ & $\begin{array}{l}\text { mean velocity components in } x, y, z \text { directions, respec- } \\
\text { tively (Fig. 1) }\end{array}$ \\
\hline$U^{*}$ & friction velocity \\
\hline$x, y, z$ & $\begin{array}{l}\text { cartesian coordinates, with } x \text { parallel to tunnel centre } \\
\text { line. } x \text { starts from the leading edge for aerofoil and } \\
\text { boundary layer data and from trailing edge for wake } \\
\text { data (Fig. 1) }\end{array}$ \\
\hline ; & boundary layer displacement thickness \\
\hline & dary layer momentum thickness \\
\hline & tematic viscosity of air \\
\hline
\end{tabular}

Subscripts

TE trailing edge

$\infty \quad$ freestream condition

$r \quad$ reference condition

Superscripts

$\langle$ root mean square value

- time averaged value

\footnotetext{
* Present address: Department of Aerospace Science Engineering, Tuskegee University, Tuskegee, AL 36088, USA
}

\section{Introduction}

The study of the characteristics of the flow field near the trailing edge of a submerged body has attracted considerable attention of research workers over the years. The free turbulent mixing process plays an important role in many practical phenomena of aerospace engineering. The flow in the near-wake of an aerofoil significantly affects the aerofoil pressure distribution and hence is of considerable interest. Wake flows form a particular class of free shear flows. A critical analysis of the available free turbulent mixing experiments and of the available models for the free turbulent shear flows is given by Harsha (1971). In the Langley conference (1972) on free turbulent shear flows, the available prediction methods for free shear flows are tested against a set of standardised data. These methods fail to predict satisfactorily the flow fields of practical interest such as those over aerofoils at moderate/ high incidence, where the presence of strong adverse pressure gradients causes rapid thickening of the boundary layers towards the trailing edge, resulting in strong viscous-inviscid interactions. The failure is attributed to the difficulties in the existing turbulence models. Recently, Marvin (1983) has reviewed the current status of turbulence modeling for external aerodynamic flows, and Patel and Scheuerer (1982) have tested the applicability of a two-equation turbulence model in 2-dimensional near and far wakes. It appears that there is still a long way to go before reliable prediction methods are developed for solving free turbulent flows of practical interest.

Considerable literature exists to throw light on the mechanism of far wake (Schlichting 1960; Townsend 1956). The concept of similarity in the far field has been well established. However, the same is not necessarily true of the near-wake field. Not only the number of studies made regarding the turbulent near wakes is limited but most of them relate to the mean flow field of near wake downstream of a flat plate. Chevray and Kovasznay (1969) made turbulence measurements in the wake of a thin flat plate. Low speed experimental studies of the 
of $4.85 \mathrm{ohms}$ at $27^{\circ} \mathrm{C}$. Each wire was operated from a DISA $55 \mathrm{M}$ constant temperature anemometer unit at a resistance of usually 1.8 times the cold resistance.

\section{Experiments}

All the measurements were made at a constant upstream reference Reynolds number (based on chord) of $10^{6}$, corresponding to an upstream velocity of $27-31 \mathrm{~m} / \mathrm{sec}$. The upstream reference station was located at a distance of $1.1 \mathrm{~m}$ from the leading edge of the model. A standard Pitot probe permanently installed at the reference station was used to monitor the tunnel speed on a precision micromanometer. Before setting up the speed, the tunnel was allowed to run for some time (usually a half-hour) to attain steady state conditions. Detailed measurements of static pressure field, two-dimensional profiles of mean velocity, turbulence intensity and Reynolds shear stress were made at preselected streamwise stations located along the mid-chord and centre-line of the wake. Wake measurements extended up to three chord lengths.

In addition to these, some measurements were made at spanwise stations to check the two-dimensionality of the flow field in the boundary layer and in the wake. Also, to check the symmetry of the profiles on either side of the trailing edge, profile measurements were carried out on both surfaces of the aerofoil close to the trailing edge. All the pressure measurements were made with a precision micromanometer. In the case of hot wire surveys, the hot wire was calibrated in the free-stream of the tunnel just before and just after each survey. When the drift in temperature was small $\left(<1^{\circ} \mathrm{C}\right)$, a mean calibration curve interpolated from the initial and final calibration curves was used to reduce the hot-wire survey data. Otherwise, the experiment was usually repeated in its entirety.

\section{Discussion of results}

The experimental data are briefly discussed below with particular emphasis on the change-over of wall turbulence to free turbulence as the boundary layers at the trailing edge of the model merge into a wake. All experimental data has been tabulated and discussed in Hebbar (1981) ${ }^{1}$. The overall experimental error in mean velocity measurements is estimated to be not more than $\pm 1 \%$. The maximum error in $\langle u\rangle^{2}$ is probably not more than $5 \%$. The maximum error in $\langle v\rangle^{2}$ may be as high as $10 \%$; a little higher in $\overline{u v}$.

\subsection{Measurements on the model}

Wall static pressure, mean and turbulence measurements on the model have been discussed at length in Hebbar

1 It was subsequently discovered that $x$-wire measurements on the aerofoil at $(x / c)=0.766$ were suspect and could not be relied upon
(1981). These measurements do not revea! any significantly new information. However, for the sake of completeness they are very briefly commented upon.

The chordwise wall static pressure distribution measured on the model at three spanwise stations indicated that the two-dimensionality of the pressure field over the mid-span was very good. The pressure distribution obtained was typical of a conventional round-nosed symmetric aerofoil in low speeds. The effect of adverse pressure gradient on the model was very clearly reflected in the flattening of the mean velocity profiles as the trailing edge was approached. The turbulence profiles showed the effect of the adverse pressure gradient on the intensity and location of turbulence peaks as the trailing was approached, i.e., the pressure gradient had the effect of increasing the magnitude of the peaks as well as shifting the location of the peaks away from the wall.

\subsection{Static pressure in the wake}

Excepting very close to the trailing edge, a conventional static pressure probe was used to measure the static pressure distribution in the wake. To minimise probe interference in the neighbourhood of the trailing edge, a disktype static probe was used at $x=3 \mathrm{~mm}$ and $x=9 \mathrm{~mm}$. The measurements indicated a slight, favourable, longitudinal pressure gradient in the near wake. Detailed discussion of static pressure distribution in the wake appears in Hebbar (1981). It suffices to mention here that small static pressure variations (spanwise and across the wake) observed very close to the trailing edge disappeared downstream of the trailing edge; the two-dimensionality of the static pressure field was quickly established in the wake with pressure distribution becoming uniform and symmetric across the wake.

\subsection{Mean flow in the wake}

Figure 2 compares the mean velocity profiles on either side of the wake at $2 \mathrm{~mm}$ downstream of the trailing edge. The symmetry of the wake is considered quite good, the maximum difference between the mean velocities on either side being less than $6 \%$. In fact, at all stations measurements were made on both sides of the wake, but for brevity measurements on one side of the wake only are reported here.

The streamwise development of the wake as measured with a boundary layer Pitot probe is presented in the form of mean velocity profiles in Fig. 3. In the near-wake region, $\left(x / \theta_{T E}\right)<\approx 50$, only the inner portion of the profile (i.e., central portion of wake) changes with downstream distance. It accelerates quite rapidly in the streamwise direction. The outer portion of the profile essentially remains unchanged from the original shape of the boundary layer profile. It is only after $\left(x / \theta_{T E}\right)=50$, the profile begins to change over to a wake-like shape. Compared to the widening of the wake behind a bluff body, the change- 


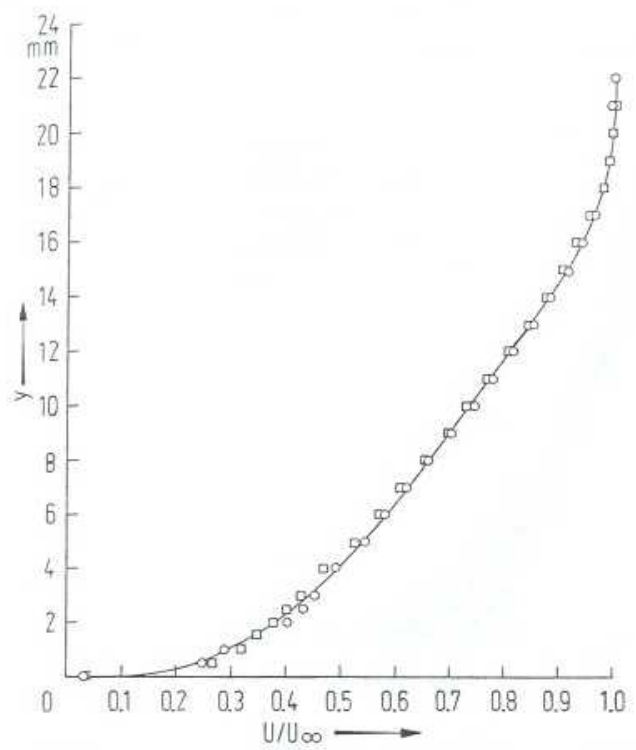

Fig. 2. Comparison of mean-velocity profiles on either side of the wake, $2 \mathrm{~mm}$ downstream of the trailing edge, $O$ and $\square$

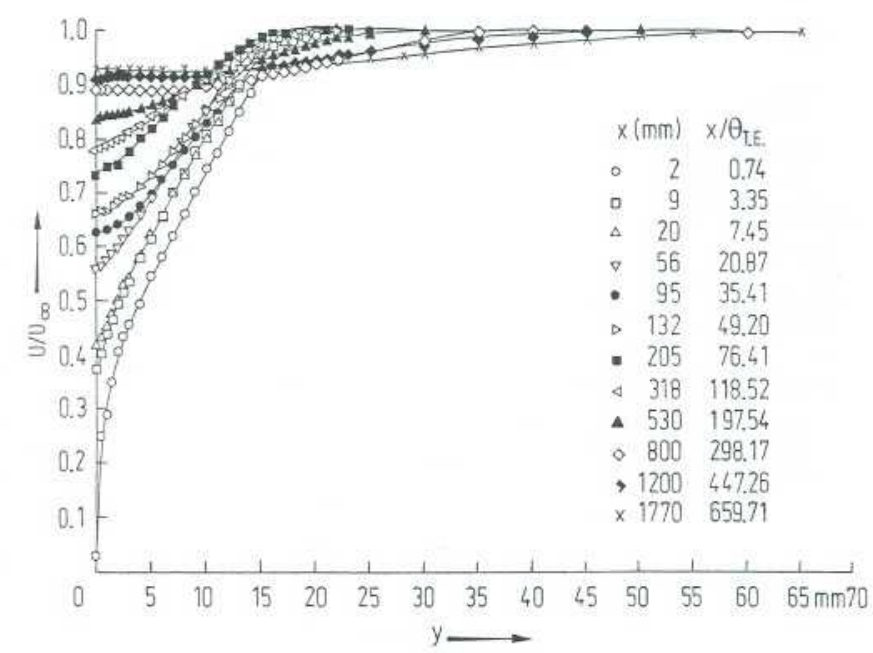

Fig. 3. Mean-velocity profiles in the wake

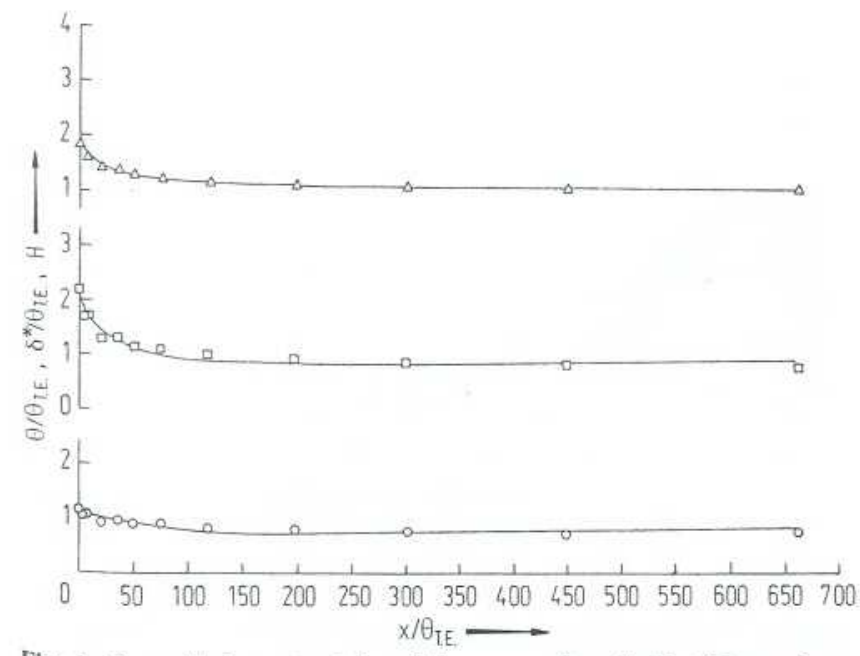

Fig. 4. Overall characteristics of the mean-flow field of the wake over here is a slow process as there is relatively little additional turbulence production at the trailing edge.

The overall characteristics of the mean-flow field of the wake are presented in Fig. 4. The displacement thickness, $\delta^{*}$, tends to approach a constant value in the far-wake region. $H$ is very large in the beginning, but begins to decrease immediately and tends to reach unity asymptotically, which is indicative of the tendency towards selfpreservation (see section 4.6). It may be noted here that for self-preservation in a two-dimensional wake $\delta^{*}$ must be constant and $H=1$. In the region close to the trailing edge $\left(0 \leq x / \theta_{T E} \leq 150\right)$, the momentum thickness decreases with downstream distance suggesting a favourable pressure gradient. This fact is of course to be expected in the very near-wake region because of the initial acceleration of the streamlines past the trailing edge. Further downstream, $\theta$ tends to approach a constant value. However, a closer look at the momentum thickness distribution (Hebbar 1981) reveals that at large distances from the trailing edge, the wake momentum thickness is indeed not settled down, but shows a slight decreasing trend. This implies a lack of two-dimensionality and suggests a favourable pressure gradient induced by flow convergence in the tunnel due to the growth of tunnel wall boundary layers.

The asymptotic analysis of turbulent wake of a thin flat plate by Alber (1980) and its comparison with the wake data of Chevray and Kovasznay (1969) has shown that the first-order center-line velocity in the near-wake region $\left(U^{*} x / v>300\right.$ and extending downstream approximately ten boundary-layer thicknesses) increases logarithmically with downstream distance. The near-wake flat-plate data of Andreopoulos and Bradshaw (1980) and Ramaprian et al. (1982) also suggest that the center-line velocity closely follows a semi-logarithmic relationship. The present investigation confirms this fact as can be seen from Fig. 5, where the center-line velocity follows a logarithmic relationship in the region $10^{3}<U^{*} x / v<4 \times 10^{4}$. However, the constants in the logarithmic relationship for

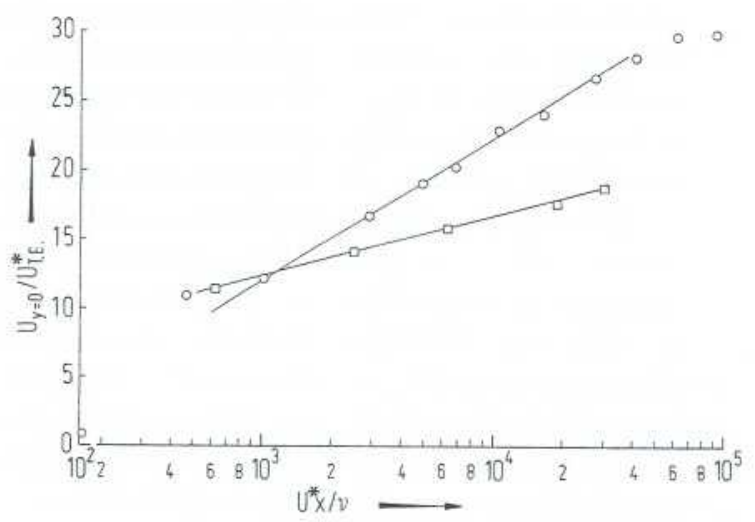

Fig. 5. Semilogarithmic relationship for the near-wake centre-line velocity; o present data; $\square$ Chevray and Kovasznay (1969) 
the present data are different from those for the flat-plate data. This is attributed to the presence of a favorable pressure gradient in the near-wake region.

The results shown here correspond to a value of the trailing-edge shape parameter $H_{T E}=1.54$ and their trend close to the trailing edge is somewhat different from that of flat-plate results reported by others for lower values of the shape parameter, viz., $H_{T E}=1.44$ by Chevray and Kovasznay (1969) and 1.283 by Agrawal et al. (1977).

Figure 6 shows the non-dimensional mean-velocity defect versus the normal distance from wake center-line normalised by the half-wake width. Also included in the figure for comparison are the Gaussian distribution of mean-velocity profiles, the Coles' wake function suitably normalised and the distribution based on the experimental data of Prabhu (1971). Though the mean-velocity defect profiles tend to approach local (geometric) similarity form quite quickly within about twenty momentum thicknesses behind the trailing edge, the downstream profiles do not collapse onto a single curve. In fact, similarity is not yet fully established even at distances of 500 trailing-edge momentum thicknesses downstream. It is suggested that the lack of two-dimensionality in the flow field referred to earlier might have probably influenced the measurements far downstream resulting in the deviation from similarity.

\subsection{Turbulence in the wake}

In what follows only the turbulence data taken with an $x$-wire is discussed. Only two components of turbulence, namely $u$ and $v$, were measured with an $x$-wire. In the absence of measured value for the third component of turbulence, $w$, the usual assumption was made to compute the turbulence energy of the flow field: $\langle w\rangle^{2}=0.75\langle u\rangle^{2}$ for boundary layer flows (Marvin 1977) and $\langle w\rangle^{2}=\langle v\rangle^{2}$ for wake flows (Harsha and Lee 1970), where \langle\rangle denotes root-mean-square value of the turbulence component.

The intensities of longitudinal (streamwise) and normal components of velocity fluctuations in the wake are shown in Figs. 7 and 8, respectively. A careful observation of the turbulence intensity variations show two distinct patterns, the dividing limit being $\left(x / \theta_{T E}\right)=50$. With the presence of characteristic peaks close to the center-line of the wake $(y=0)$, the intensity profiles very close to the trailing edge $\left(x / \theta_{T E}<50\right)$ resemble those typical of a two-dimensional boundary layer. The peaky characteristic of the profiles disappears at large distances from the trailing edge $\left(x / \theta_{T E}>50\right)$. Thus, the data of the present experiments, which correspond to $H_{T E}=1.54$, suggest $\left(x / \theta_{T E}\right)=50$ as the limit at which the wake structure changes from the near-wake type (wall turbulence) to the far-wake type (free turbulence). The dividing limits reported by Chevray and Kovasznay (1969) and Agrawal et al. (1977) are 30 for $H_{T E}=1.44$ and 20 for $H_{T E}=1.283$, respectively.

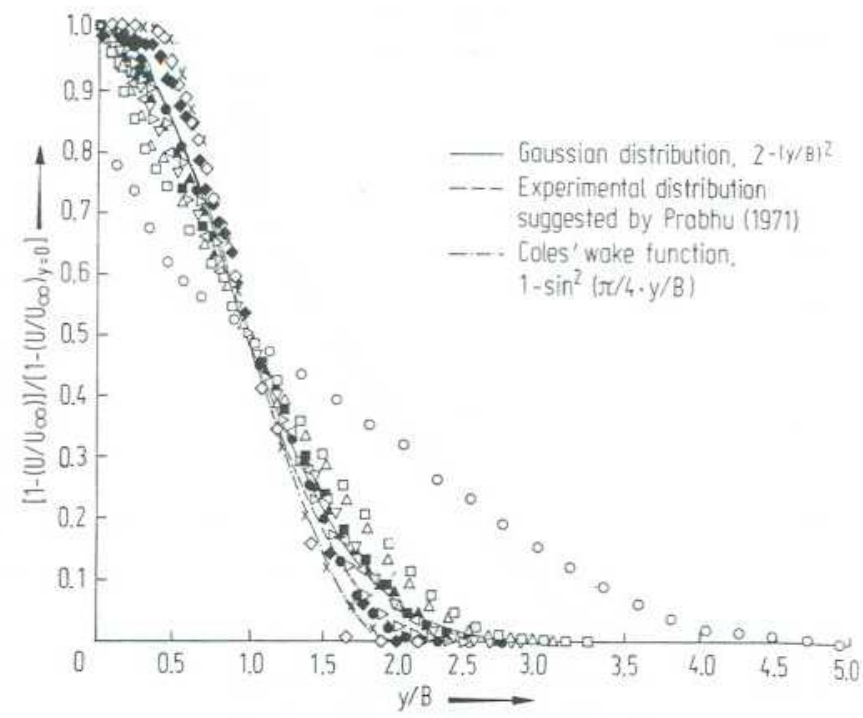

Fig. 6. Non-dimensional mean-velocity defect distribution in the wake; symbols see Fig. 3

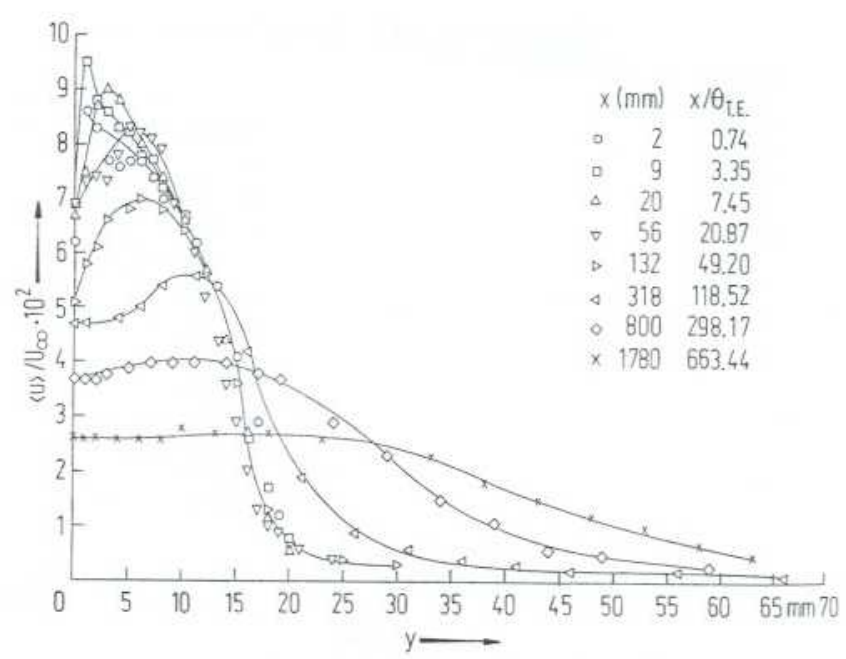

Fig. 7. Longitudinal turbulence intensity profiles in the wake

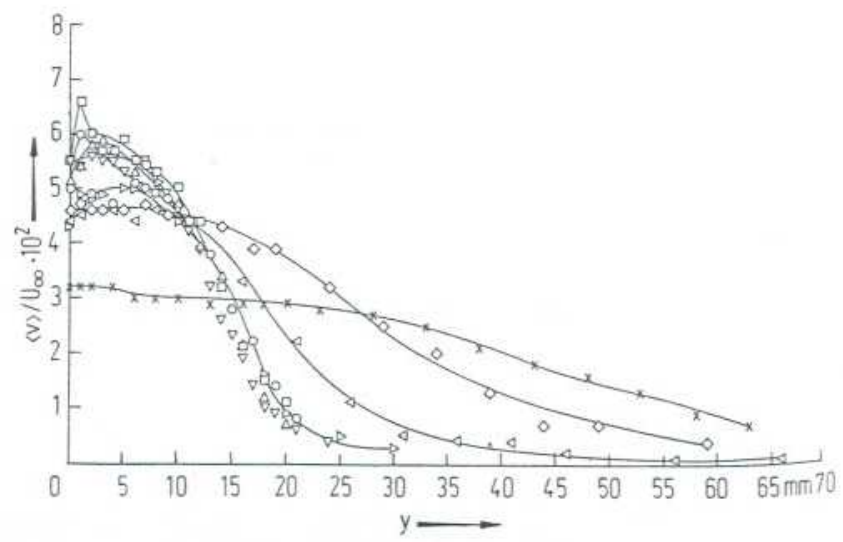

Fig. 8. Normal turbulence intensity profiles in the wake; symbols see Fig. 7 


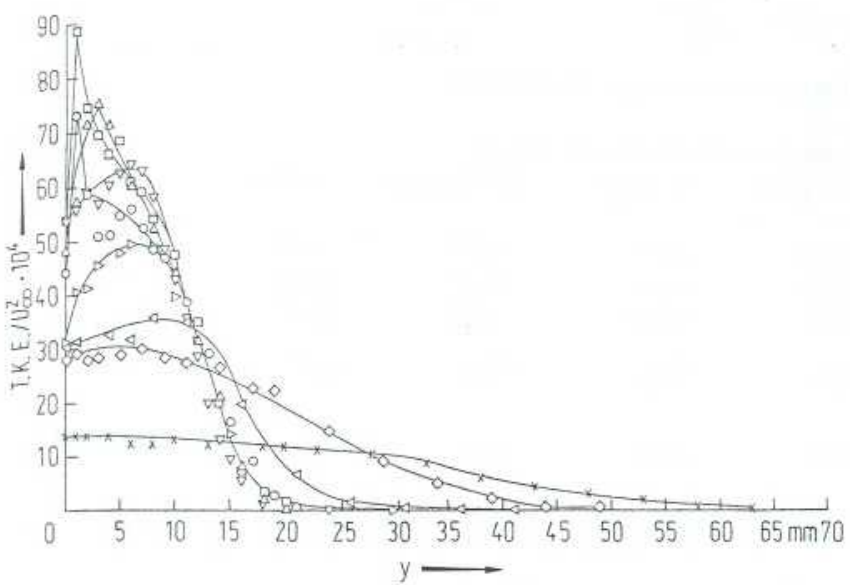

Fig. 9. Turbulent kinetic energy profiles in the wake; symbols see Fig. 7

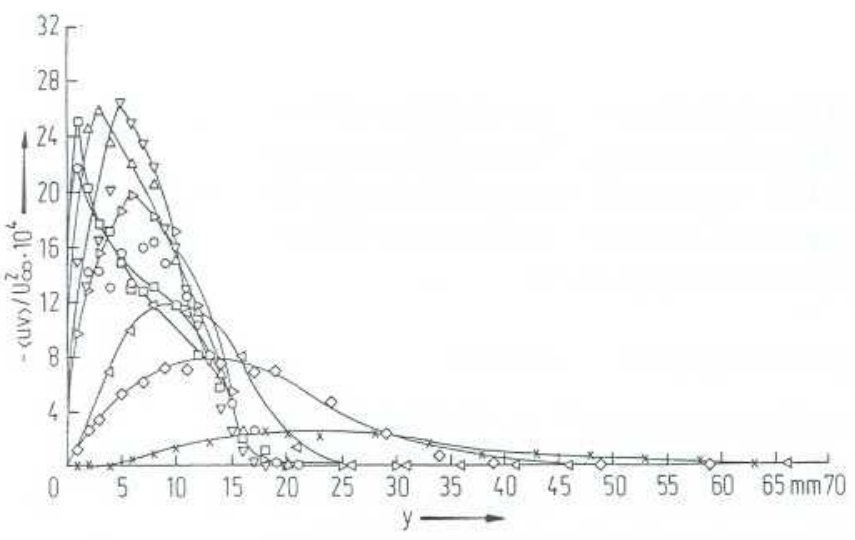

Fig. 10. Reynolds shear stress profiles in the wake; symbols see Fig. 7

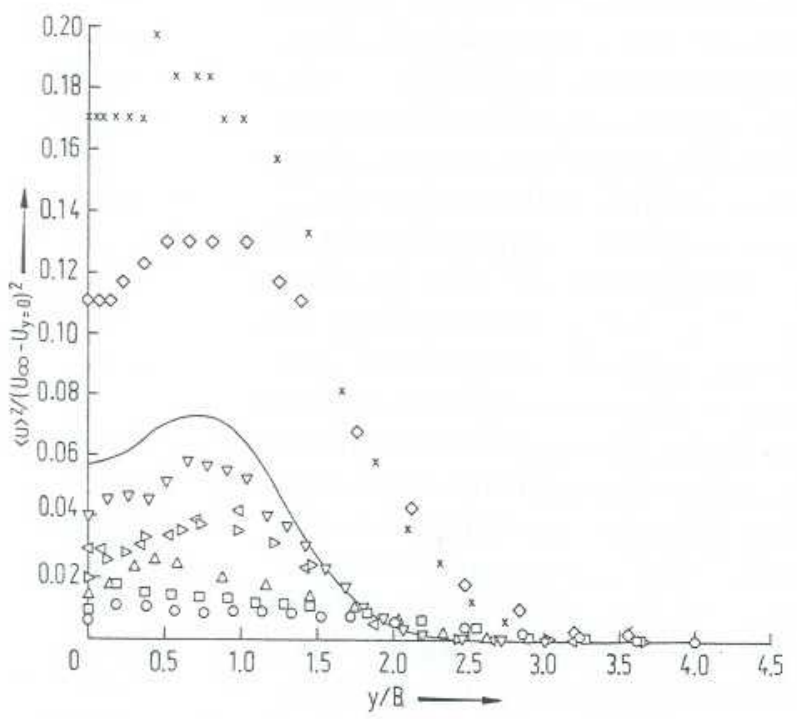

Fig. 11. Mean square longitudinal turbulence intensity distribution across the wake normalized by mean-flow scales; symbols see Fig. $7 ;-$ : equilibrium distribution suggested by Prabhu (1971)

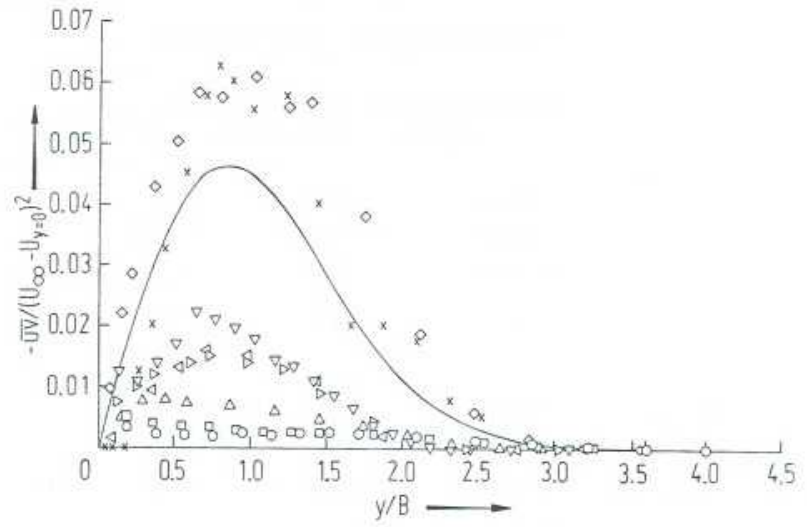

Fig. 12. Reynolds shear stress distribution across the wake nornalized by mean-flow scales; symbols see Fig. 7 ; — : equilibrium distribution based on Gaussian velocity profile

The change-over of wall turbulence to free turbulence as the boundary layers at the trailing edge of the model merge into a wake is very clearly seen in the restructuring of turbulence intensity profiles. Very close to the trailing edge, the longitudinal intensity is roughly $1 \frac{1}{2}$ times the normal intensity, which is expected there because of the restraining effect of the wall on normal component. However, this effect decreases further away from the trailing edge, and at large distances from the trailing edge, the longitudinal and normal intensities become more nearly equal (Figs. 7 and 8 ).

The distributions of turbulence kinetic energy and Reynolds shear stress in the wake are shown in Figs. 9 and 10 , respectively. The trend of the curves shown in Fig. 9 follows closely that of the curves of Fig. 7. The correlation between turbulent shear stress and turbulent kinetic energy in the wake (not shown here) may be satisfactorily represented by a straight line passing through the origin with a slope of 0.295 which may be compared with an average value of 0.3 suggested by Harsha and Lee (1970) based on a detailed study of a large amount of turbulent shear stress and kinetic energy data in incompressible flows.

It is known that the second order correlations of velocity fluctuations approach similarity significantly later than the mean-velocity profiles (Langley Conference 1972). Because the mean flow in the wake shows deviation from similarity (see section 4.3), the profiles of turbulence intensity and Reynolds shear stress may not be expected to approach similarity within the range covered in the present investigation. This is clearly brought out in Figs. 11 and 12 , which show the distributions of mean square longitudinal turbulence intensity and Reynolds shear stress across the wake, respectively, using mean-flow scales for normalization. There is no tendency towards similarity at all in these distributions. 
Table 1. Mean-flow and turbulence parameters in the neighbourhood of trailing edge

\begin{tabular}{|c|c|c|c|c|c|c|c|c|}
\hline \multirow[t]{2}{*}{ Parameters } & \multicolumn{3}{|c|}{ Measured values on the aerofoil } & \multicolumn{5}{|c|}{ Measured values in the half wake } \\
\hline & $\begin{array}{l}\text { Distance fro } \\
(x / c)=0.85\end{array}$ & $\begin{array}{l}\mathrm{n} \text { the leading } \\
(x / c)=0.958\end{array}$ & $\begin{array}{l}\text { edge } \\
\qquad(x / c)^{a}=0.996\end{array}$ & $\begin{array}{l}\text { Distance } \mathrm{f} \\
x=2 \mathrm{~mm}\end{array}$ & $\begin{array}{l}\mathrm{n} \text { the trailin } \\
x=9 \mathrm{~mm}\end{array}$ & $\begin{array}{l}\text { dge } \\
x=20 \mathrm{~mm}\end{array}$ & $x=56 \mathrm{~mm}$ & $x=132 \mathrm{~mm}$ \\
\hline$\delta^{*}(\mathrm{~mm})$ & 2.75 & 3.64 & 4.13 & 5.85 & 4.55 & 4.57 & 3.49 & 3.12 \\
\hline$\theta(\mathrm{mm})$ & 1.85 & 2.36 & 2.68 & 3.13 & 2.81 & 2.87 & 2.45 & 2.37 \\
\hline$H$ & 1.49 & 1.54 & 1.54 & 1.87 & 1.62 & 1.59 & 1.43 & 1.32 \\
\hline $\operatorname{Max} \frac{\langle u\rangle}{U_{\infty}} 10^{2}$ & 7.0 & 7.4 & 7.7 & 8.6 & 9.5 & 9.0 & 8.3 & 7.0 \\
\hline $\operatorname{Max} \frac{\langle v\rangle}{U_{\infty}} 10^{2}$ & 4.5 & 5.0 & 5.1 & 6.0 & 6.6 & 5.9 & 5.6 & 5.1 \\
\hline $\operatorname{Max} \frac{T K E}{U_{\infty}^{2}} 10^{4}$ & 53.1 & 60.2 & 64.3 & 72.98 & 88.68 & 75.31 & 63.87 & 49.5 \\
\hline $\operatorname{Max} \frac{-\overline{u v}}{U_{\infty}^{2}} 10^{4}$ & 10.6 & 12.9 & 12.4 & 21.7 & 25.1 & 25.9 & 26.5 & 19.8 \\
\hline
\end{tabular}

a Corresponds to the location that is $2 \mathrm{~mm}$ upstream from the trailing edge

\subsection{Flow field immediately behind the trailing edge}

Table 1 lists the experimentally determined mean-flow and turbulence parameters of the flow field in the neighbourhood of the trailing edge and is intended to highlight the significant changes that take place in that region. The trend in the variation of these parameters is, of course, expected from the shape of the mean velocity and turbulence profiles in that region.

The presence of a finite mean velocity (Fig. 3) and turbulence intensities (Figs. 7 and 8) on the wake centre-line at the point of measurement that could be probed closest to the trailing edge (i.e., $2 \mathrm{~mm}$ from it) indicates rapid vortical interaction of the wall layers immediately behind the trailing edge. It is this rapid interaction process that causes significant changes in the flow parameters close to the trailing edge. In fact, a careful observation of the streamwise development of mean-flow characteristics (Table 1) and turbulence profiles (Figs. 7-10) reveals that the interaction of the wall layers is so severe that the mean-flow parameters $\delta^{*}, \theta$, and $H$, and the maximum values of turbulence intensities and Reynolds shear stress continue to grow immediately downstream of the trailing edge, before they finally begin to relax. This leads to the appearance of 'overshoot' in the profiles.

According to Ramaprian et al. (1982), who also observed similar overshoots in their experimental data, these overshoots are primarily associated with flow separation or vortex shedding behind a trailing edge of finite thickness. It is suspected that in the present case a combination of factors such as the convergence of the boundary layers at the trailing edge (which has an included angle of $19^{\circ}$ ), the presence of adverse pressure gradient and a small but nonetheless finite thickness (of magnitude 80 in wall coordinates) at the trailing edge causes rapid interaction of normal fluctuating components of the boundary layers on either side of the trailing edge. The sudden interaction results in extra production of Reynolds stress (both shear and normal) and therefore in the overshoot of wake profiles to be followed by subsequent relaxation of the wake.

\subsection{Approach to equilibrium}

At large distances from the trailing edge, i.e., in the farwake region, the structure of both the mean-flow and turbulence field is expected to reach a state of selfpreservation (equilibrium) asymptotically. The term selfpreservation (equilibrium) is used here in the sense of Townsend (1956). As already discussed, the mean flow parameters (section 4.3) and turbulence parameters (section 4.4) do not attain (local) similarity within the range covered in the present investigation, i.e., three chord lengths downstream of the trailing edge $\left(0<x / \theta_{T E}<660\right)$.

The approach to equilibrium is examined in terms of equilibrium parameters for two-dimensional wakes defined by Prabhu (1971) and recently by Sreenivasan and Narasimha (1982). Figures 13 and 14 illustrate the streamwise development of the parameters $\left(B^{2} / x \theta\right)$ and $\langle u\rangle_{\max } /\left(U_{\infty}-U_{y=0}\right)$, characterising wake thickness and turbulence intensity, respectively. Also included in the figures for comparison are the experimental data of other investigators. These parameters should ultimately become independent of downstream distance in equilibrium flows, the equilibrium values being 0.089 and 0.27 , respectively (Prabhu 1971). Sreenivasan and Narasimhä (1982) quote a value of 0.3 for $B /(x \theta)^{1 / 2}$. It is clear from these figures (also see Figs. 11 and 12 where solid lines denote equilibrium distributions) that both the mean flow and the fluctuating field have not attained self-preservation (equilibrium) even at the last measuring station. This deviation from equilibrium is again attributed to the lack 


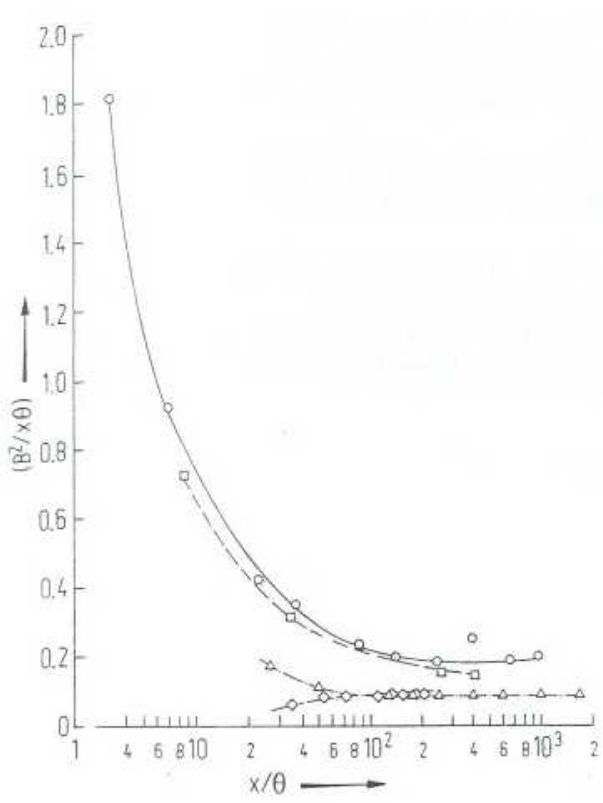

Fig. 13. Approach to equilibrium: Streamwise development of wake-thickness parameter, $\left(B^{2} / x \theta\right) ; 0$ present data; $\square$ Chevray and Kovasznay (1969); $\triangle$ Prabhu (1971); $\diamond$ Townsend (1956)

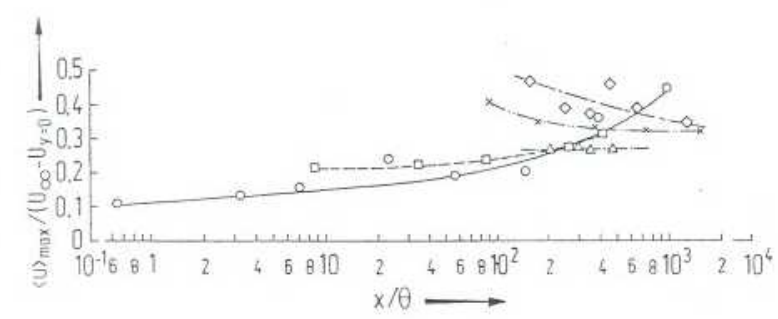

Fig. 14. Approach to equilibrium: Streamwise development of wake turbulence-intensity parameter, $\langle u\rangle_{\max } /\left(U_{\infty}-U_{y}=0\right)$; 0 present data; $\square$ Chevray and Kovasznay (1969); $\triangle$ Prabhu (1971); $\diamond$ Townsend (1956); $\times$ Uberoi and Freymuth (1969)

of two-dimensionality in the flow field far downstream (see section 4.3). It should be noted here that the data of Chevray and Kovasznay (1969) also do not indicate selfpreservation.

\section{Conclusion}

A set of mean and turbulence measurements have been carried out in the boundary layer and wake of a symmetric aerofoil in low speed flow. The experimental data have been analysed with particular emphasis on the changeover of wall-turbulence to free-turbulence as the boundary layers at the trailing edge of the model merge into a wake. The results of this study correspond to a value of the trailing-edge shape parameter $H_{\mathrm{TE}}=1.54$ and their trend close to the trailing edge is somewhat different from that of flat-plate results reported by other investigators.' The important findinge of the present study are summarized below:

(i) The rapid vortical interaction of the boundary layers immediately behind the trailing edge leads to significant changes in the flow parameters close to the trailing edge. The relaxation of the wake is preceded by initial 'overshoot' in the streamwise profiles of mean-flow parameters and peak values of turbulence components.

(ii) The inner portion of the half-wake profile (i.e., the central portion of the wake) reacts quickly to the sudden disappearance of wall shear stress at the trailing edge, whereas the outer portion of the profile essentially remains unchanged from the original shape of the boundary layer profile. The profile begins to change over to a wake-like shape only after $\left(x / \theta_{T E}\right) \simeq 50$, which may be considered as the dividing limit between the near-wake (wall turbulent flow) and far-wake (free turbulent flow) regions.

(iii) The inner layer turbulence reacts quickly to changes in wall boundary conditions (i.e., to the effect of the absence of a solid boundary downstream of the trailing edge). As the boundary layers at the trailing edge of the model merge and subsequently grow into a wake, two distinct patterns appear in the turbulence structure with $\left(x / \theta_{T E}\right) \simeq 50$ as the dividing limit where the structure changes from wall-turbulence type to free-turbulence type.

(iv) Both the mean flow field and the fluctuating field do not exhibit any tendency towards similarity/equilibrium within the range covered in the experiments.

\section{Acknowledgements}

The author offers his sincere thanks to the staff members of the Boundary Layer Laboratory of the Aerodynamics Division for their contributions to various phases of this investigation. The author gratefully acknowledges the useful suggestions of Prof. R. Narasimha and Dr. K. S. Yajnik. Finally, permission of the Director of NAL for publishing this work is acknowledged.

\section{References}

Agrawal, M. L.; Pande, P. K.; Prakash, R. 1977: Study of the turbulent near wake of a flat plate. AIAA 3. 15, 740-743

Alber. I. E. 1980: Turbulent wake of a thin flat plate. AIAA 1 18, 1044-1051

Andreopoulos. J.; Bradshaw, P. 1980: Measurements of interacting turbulent shear layers in the near wake of a flat plate. 3. Fluid Mech. 100, 639-668

Chevrav, R.; Kovasznay, L. S. G. 1969: Turbulence measurements in the wake of a thin flat plate. AIAA 3. 7, 1641-1643

Harsha. P. T. 1971: Free turbulent mixing: A critical evaluation of theory and experiment. AEDC-TR-71-36

Harsha, P. T.; Lee. S. C. 1970: Correlation between turbulent shear stress and turbulent kinetic energy. AIAA J. 8, 1508-1510 
Hebbar, K. S. 1981: Experimental study of incompressible mean and turbulence flow fields on and behind a symmetric aerofoil. Technical Memorandum AE-TM-4-81, National Aeronautical Laboratory, Bangalore, India

Langley Conference 1972: Free turbulent shear flows, Vol. I Conference proceedings. NASA SP-321

Marvin, J. G. 1977: Turbulence modeling for compressible flows. NASA TAM-73, 188

Marvin. J. G. 1983: Turbulence modeling for computational aerodynamics. ALAA J. 21, 941-955

Patel. V. C.: Scheuerer. G. 1982: Calculation of two-dimensional near and far wakes. AIAA J. 20. 900-907

Prabhu. A. 1971: Nonequilibrium wake flows. Ph.D. thesis, Indian Institute of Science, Bangalore, India
Ramaprian, B. R.: Patel, V. C.:: Sastry, M. S. 1982: The symmetric turbulent wake of a flat plate. AIAA J. 20, 1228-123

Schlichting, H. 1960: Boundary layer theory. New York: McGrawHill

Sreenivasan, K. R.: Narasimha, R. 1982: Equilibrium parameters for two-dimensional turbulent wakes. J. Fluids Eng. 104, $167-170$

Townsend, A. A. 1956: The structure of turbulent shear flow, London: Cambridge University Press

Ubcroi, M. S.: Freymuth, P. 1969: Spectra of turbulence in wakes behind circular cylinders. Phys. Fluids 12, 1359-1363

Received July 22, 1985 